\title{
Analysis of Repairable Geo/G/1 Queues with Negative Customers
}

\author{
Doo Ho Lee ${ }^{1}$ and Kilhwan Kim ${ }^{2}$ \\ ${ }^{1}$ Software Contents Research Lab, ETRI, Daejeon 305-700, Republic of Korea \\ ${ }^{2}$ Department of Management Engineering, Sangmyung University, Chungnam 330-720, Republic of Korea \\ Correspondence should be addressed to Doo Ho Lee; enjdhlee@gmail.com
}

Received 3 September 2014; Accepted 8 December 2014; Published 24 December 2014

Academic Editor: Kannan Krithivasan

Copyright (c) 2014 D. H. Lee and K. Kim. This is an open access article distributed under the Creative Commons Attribution License, which permits unrestricted use, distribution, and reproduction in any medium, provided the original work is properly cited.

\begin{abstract}
We consider discrete-time Geo/G/1 queues with negative customers and a repairable server. The server is subject to failure due to a negative customer arrival. As soon as a negative customer arrives at a system, the server fails and one positive (ordinary) customer is forced to leave. At a failure instant, the server is turned off and the repair process immediately begins. We construct the mathematical model and present the probability generating functions of the system size distribution and the FCFS sojourn time distribution. Finally, some numerical examples are given to show the influence of negative customer arrival on the performance measures of the system.
\end{abstract}

\section{Introduction}

After the introduction in the work of Gelenbe [1], there has been a rapid increase in the literature on queueing systems with negative customers due to their applications to neural networks, communication systems, and manufacturing systems. If a negative customer arrives at a queueing system, it removes one ordinary customer (called a positive customer) according to a predetermined removal discipline. There are two typical removal disciplines: (i) a negative customer removes the customer being served $(\mathrm{RCH})$; (ii) a negative customer removes the customer who arrived most recently (RCE). The negative customers cannot accumulate in the queue and do not receive services. The negative customers have a rich and wide range of real applications such as job canceling signals accompanied by machine breakdowns [2], network routing control [3], and load balancing [4]. For example, if a certain virus enters a computer system, the virus interrupts system operations, deletes files, and infects other files. In telecommunication systems, the negative customers can be used to model cell losses caused by the arrival of a corrupted cell, when the preceding cells of a packet would be discarded.

The concept of the negative customer has been applied to the $\mathrm{M} / \mathrm{M} / 1$ queue [5], the $\mathrm{M} / \mathrm{G} / 1$ queue [6], and the GI/M/1 queue [7]. Excellent surveys on negative customers have been provided by Artalejo [8], Do [9, 10], and Gelenbe [11-13].
Recently, this topic has been extended to a discrete-time queueing system. Note that the discrete-time queue is more suitable to describe the operation of time-slotted digital communication systems due to the packetized nature of transport protocols. Atencia and Moreno [14] studied the $\mathrm{Geo} / \mathrm{Geo} / 1$ queues with negative customers and various removal disciplines caused by the negative customers. Zhou [15] investigated the discrete-time GI/G/1 queue with RCHtype negative customers. Wang and Zhang [16] considered the $\mathrm{Geo} / \mathrm{Geo} / 1$ retrial queue with negative customers and an unreliable server. Park et al. [17] obtained the results of the Geo/G/1 queue with negative customers and without repair times. Recently, Chae et al. [18] extended Atencia and Moreno's [14] Geo/Geo/1 queues to the GI/Geo/1 queues, where the interarrival times are generally distributed. The results of the queueing system with negative customers under the assumption of a Markovian arrival process can be found in $\mathrm{Wu}$ et al. $[19,20]$.

In most of the literature on queueing systems with negative customers, the server is assumed to be reliable on a permanent basis, regardless of the arrivals of negative customers. However, in many practical systems, the arrival of a negative customer can cause server breakdown as well as destruction of work in a system. For example, when a computer server is infected with a computer virus, a recovery time is needed 
to restore the server. Similarly, when a canceling signal for the job in progress arrives at a database server, a system cleaning time such as a rollback time for incomplete data operation may be needed. For this reason, we consider the Geo/G/1 queues with negative customers under the assumption that the server is repairable. Since the performance measure of such a system may be influenced by breakdown and repair time, the repairable system is well worth analyzing in view of queueing theory along with reliability theory.

There are few researches on repairable queueing systems with negative customers. Wang and Zhang [16] considered the $\mathrm{Geo} / \mathrm{Geo} / 1$ retrial queue with negative customers and an unreliable server, where server breakdown occurs whenever negative customers arrive at a system. Lee et al. [21] presented the results of repairable $\mathrm{Geo} / \mathrm{G} / 1$ queues with disasters, where if a disaster arrives at a system, all present customers (i.e., a customer in service plus customers in queue) are forced to leave the system. In [21], the authors considered a disaster as server failure which leads to the destruction of all work in process in a system. However, neither of them considered the case of repairable queueing systems with negative customers which cause server breakdown as well as canceling a single ordinary customer.

Systems under our study have following features. Positive customers arrive at a single server queue, according to a Bernoulli process. A server provides a service to each customer on an FCFS basis. The service times are independent and identically distributed (i.i.d.) random variables that are generally distributed. Negative customers arrive when the server is busy according to a Bernoulli process. Negative customers do not have an effect on the system if the server is idle or under repair. Each time a negative customer arrives at the system while the server is busy, the server fails and the customer in service leaves the system. At a failure epoch, the server is turned off and a repair period immediately begins. The server repair times are i.i.d. random variables that are generally distributed. During the repair period, the stream of new arrivals continues. Depending on whether new arrivals are allowed to enter the system during the repair time, there can be one of the two following systems. In System 1, the customers newly arriving during the repair period cannot enter the system and are blocked. This is the case when a virus-infected computer server is isolated from the network during the repair time without any backup server. In System 2, they join the queue and wait for the server to be repaired. This is the case when a backup server just stores requests during the repair time and passes them to the original server when it is recovered. As soon as the repair period ends, the server promptly becomes available. Both Systems 1 and 2 have RCH discipline. For each system, using the probability generating function (PGF) technique, we present PGFs of the system size distribution and the sojourn time distribution.

In this paper, we first describe the mathematical model in Section 2. In Sections 3 and 4, general results on the system size and the sojourn time of System 1 and System 2 are presented. Section 5 deals with numerical experiments which we conducted to investigate the influence of the arrival of negative customers on the mean system size of each system.

\section{Model Description}

Throughout this paper, we adopt the late arrival system (LAS) [22]. Let the time axis be marked by $t=0,1,2, \ldots$. According to the LAS model, the potential arrival of a positive customer takes place during the interval $\left(t^{-}, t\right)$ and the potential service completion occurs during the interval $\left(t, t^{+}\right)$, where $t^{+}$and $t^{-}$ represent $\lim _{\Delta t \rightarrow 0}(t+|\Delta t|)$ and $\lim _{\Delta t \rightarrow 0}(t-|\Delta t|)$, respectively. Since the arrivals of a positive customer and a negative customer can occur simultaneously at a slot boundary, the order of these events must be stated. We assume that the potential arrival of a negative customer occurs during the interval $\left(t^{-}, t\right)$ and immediately before the potential arrival of a positive customer. We also assume that the potential completion of a repair time occurs during the interval $\left(t^{-}, t\right)$ and immediately before the potential arrival of a positive customer. We further assume that a negative customer arrival and a repair completion do not occur at the same slot boundary simultaneously.

We define commonly used notations to analyze both Systems 1 and 2. Interarrival times of positive customers $\left\{A_{n}\right\}_{n=1}^{\infty}$ are i.i.d. discrete random variables and follow a geometric distribution with parameter $\lambda$ :

$$
\begin{aligned}
\operatorname{Pr}\left\{A_{n}=k\right\} & =\bar{\lambda}^{k-1} \lambda, \quad k \geq 1 ; \\
\bar{\lambda} & =1-\lambda, \quad 0<\lambda<1 ; \\
E\left[A_{n}\right] & =\lambda^{-1} .
\end{aligned}
$$

Interarrival times of negative customers $\left\{Y_{n}\right\}_{n=1}^{\infty}$ are i.i.d. discrete random variables and follow a geometric distribution with parameter $\eta$ :

$$
\begin{aligned}
& \operatorname{Pr}\left\{Y_{n}=k\right\}=\bar{\eta}^{k-1} \eta, \quad k \geq 1 ; \\
& \bar{\eta}=1-\eta, \quad 0<\eta<1 ; \\
& E\left[Y_{n}\right]=\eta^{-1} \text {. }
\end{aligned}
$$

Service times $\left\{S_{n}\right\}_{n=1}^{\infty}$ are i.i.d. general discrete random variables. A distribution and its PGF are denoted by $\operatorname{Pr}\left\{S_{n}=k\right\}=$ $s_{k}(k \geq 1)$ and $S(z)=\sum_{k=1}^{\infty} s_{k} z^{k}$, respectively. Repair times $\left\{R_{n}\right\}_{n=1}^{\infty}$ are i.i.d. general discrete random variables and have the distribution $\operatorname{Pr}\left\{R_{n}=k\right\}=r_{k}(k \geq 1)$ and its PGF $R(z)=\sum_{k=1}^{\infty} r_{k} z^{k}$. We assume that $\left\{A_{n}\right\}_{n=1}^{\infty},\left\{Y_{n}\right\}_{n=1}^{\infty},\left\{S_{n}\right\}_{n=1}^{\infty}$, and $\left\{R_{n}\right\}_{n=1}^{\infty}$ are mutually independent.

Systems 1 and 2 are represented by a Markov chain. Let $N\left(t^{+}\right)$be the number of customers in the system at $t^{+}$. Let $\xi\left(t^{+}\right)$be the server state at $t^{+}$and be defined as follows:

$$
\xi\left(t^{+}\right)= \begin{cases}0, & \text { The server is under repair at } t^{+} \\ 1, & \text { The server is available at } t^{+}\end{cases}
$$

Then, in both System 1 and System $2,\left\{N\left(t^{+}\right), \xi\left(t^{+}\right), S_{R}\left(t^{+}\right)\right.$, $\left.R_{R}\left(t^{+}\right), t=0,1, \ldots\right\}$ is a Markov chain, where the supplementary variables $S_{R}\left(t^{+}\right)$and $R_{R}\left(t^{+}\right)$, respectively, represent the remaining service time and the remaining repair time all 
at $t^{+}$. One of our main purposes is to obtain the stationary distribution:

$$
P_{n}=\lim _{t \rightarrow \infty} \operatorname{Pr}\left\{N\left(t^{+}\right)=n\right\}, \quad n \geq 0 .
$$

Since we are interested in the steady-state behavior of the systems, we employ the following limiting probabilities throughout this paper to manipulate the Kolmogorov equations of the Markov chain:

$$
\begin{aligned}
& \psi_{n}(k)=\lim _{t \rightarrow \infty} \operatorname{Pr}\left\{N\left(t^{+}\right)=n, \xi\left(t^{+}\right)=0, R_{R}\left(t^{+}\right)=k\right\}, \\
& n \geq 0, \quad k \geq 1 \text {, } \\
& \pi_{n}(k)=\lim _{t \rightarrow \infty} \operatorname{Pr}\left\{N\left(t^{+}\right)=n, \xi\left(t^{+}\right)=1, S_{R}\left(t^{+}\right)=k\right\}, \\
& n \geq 1, k \geq 1 \text {, } \\
& \psi_{n}=\lim _{t \rightarrow \infty} \operatorname{Pr}\left\{N\left(t^{+}\right)=n, \xi\left(t^{+}\right)=0\right\}=\sum_{k=1}^{\infty} \psi_{n}(k), \\
& n \geq 0, \\
& \pi_{0}=\lim _{t \rightarrow \infty} \operatorname{Pr}\left\{N\left(t^{+}\right)=0, \xi\left(t^{+}\right)=1\right\}, \\
& \pi_{n}=\lim _{t \rightarrow \infty} \operatorname{Pr}\left\{N\left(t^{+}\right)=n, \xi\left(t^{+}\right)=1\right\}=\sum_{k=1}^{\infty} \pi_{n}(k), \\
& n \geq 1 \text {. }
\end{aligned}
$$

To solve the Kolmogorov equations, we introduce PGFs:

$$
\begin{gathered}
\Psi(z, k)=\sum_{n=0}^{\infty} \psi_{n}(k) z^{n}, \quad|z| \leq 1, \\
\Pi(z, k)=\sum_{n=1}^{\infty} \pi_{n}(k) z^{n}, \quad|z| \leq 1, \\
\Psi^{*}(z, w)=\sum_{k=1}^{\infty} \Psi(z, k) w^{k}, \quad|w| \leq 1, \\
\Pi^{*}(z, w)=\sum_{k=1}^{\infty} \Pi(z, k) w^{k}, \quad|w| \leq 1 .
\end{gathered}
$$

\section{System 1}

In System 1, newly arriving customers are blocked when the server is under repair.

3.1. System Size Distribution for System 1. With the limiting probabilities defined in Section 2, the Kolmogorov equations for the stationary distribution are given by

$$
\begin{gathered}
\psi_{n}(k)=\psi_{n}(k+1)+\eta \pi_{n+1} r_{k}, \quad n \geq 0, \\
\pi_{0}=\left(\psi_{0}(1)+\pi_{0}+\bar{\eta} \pi_{1}(1)\right) \bar{\lambda}, \\
\pi_{1}(k)=\psi_{0}(1) \lambda s_{k}+\psi_{1}(1) \bar{\lambda} s_{k}+\pi_{0} \lambda s_{k} \\
+\bar{\eta}\left(\pi_{1}(1) \lambda s_{k}+\pi_{1}(k+1) \bar{\lambda}+\pi_{2}(1) \bar{\lambda} s_{k}\right),
\end{gathered}
$$

$$
\begin{aligned}
\pi_{n}(k)= & \psi_{n-1}(1) \lambda s_{k}+\psi_{n}(1) \bar{\lambda} s_{k} \\
& +\bar{\eta}\left(\pi_{n-1}(k+1) \lambda+\pi_{n}(1) \lambda s_{k}\right. \\
& \left.\quad+\pi_{n}(k+1) \bar{\lambda}+\pi_{n+1}(1) \bar{\lambda} s_{k}\right), \quad n \geq 2,
\end{aligned}
$$

and the normalizing condition is given by

$$
\pi_{0}+\Psi^{*}(1,1)+\Pi^{*}(1,1)=1 .
$$

Multiplying (7) by $z^{n}$ and summing over $n, n \geq 0$, we obtain

$$
\Psi(z, k)=\Psi(z, k+1)+\eta r_{k} z^{-1} \Pi^{*}(z, 1) .
$$

Multiplying (12) by $w^{k}$ and summing over $k, k \geq 1$, yield

$$
\Psi^{*}(z, w)\left(1-w^{-1}\right)=\eta z^{-1} R(w) \Pi^{*}(z, 1)-\Psi(z, 1) .
$$

To obtain $\Psi(z, 1)$, we insert $w=1$ into (13). Thus,

$$
\Psi(z, 1)=\eta z^{-1} \Pi^{*}(z, 1) .
$$

Incorporating (14) back into (13) then yields

$$
\Psi^{*}(z, w)=\frac{\eta w(R(w)-1)}{z(w-1)} \Pi^{*}(z, 1) .
$$

Letting $w=1$ in (15), we obtain the following relationship by the L'Hospital rule:

$$
\Psi^{*}(z, 1)=\eta E[R] z^{-1} \Pi^{*}(z, 1) .
$$

Multiplying (9) and (10) by $z^{n}$ and then summing over $n$, $n \geq 1$, together with (8), we obtain

$$
\begin{aligned}
& \Pi(z, k) \\
& \quad=\omega_{1} \Pi(z, k+1) \\
& \quad+s_{k}\left[\omega_{0} \Psi(z, 1)+z^{-1} \omega_{1} \Pi(z, 1)-\pi_{0} \lambda(1-z)\right],
\end{aligned}
$$

where $\omega_{0}=\bar{\lambda}+\lambda z$ and $\omega_{1}=\bar{\eta}(\bar{\lambda}+\lambda z)$. Multiplying (17) by $w^{k}$ and summing over $k, k \geq 1$, yield

$$
\begin{aligned}
\Pi^{*}(z, w) & \left(1-w^{-1} \omega_{1}\right) \\
= & z^{-1} \omega_{1} \Pi(z, 1)(S(w)-z) \\
& \quad+S(w)\left[\omega_{0} \Psi(z, 1)-\pi_{0} \lambda(1-z)\right]
\end{aligned}
$$

Inserting $w=\omega_{1}$ into (18) and solving $\Pi(z, 1)$, we obtain

$$
\Pi(z, 1)=\frac{z S\left(\omega_{1}\right)\left[\pi_{0} \lambda(1-z)-\omega_{0} \Psi(z, 1)\right]}{\omega_{1}\left(S\left(\omega_{1}\right)-z\right)} .
$$

Utilizing (14) and (19) in (18), $\Pi^{*}(z, w)$ is represented as

$$
\begin{aligned}
& \Pi^{*}(z, w) \\
& \quad=\frac{w\left(S(w)-S\left(\omega_{1}\right)\right)\left[\pi_{0} \lambda z(1-z)-\eta \omega_{0} \Pi^{*}(z, 1)\right]}{\left(w-\omega_{1}\right)\left(S\left(\omega_{1}\right)-z\right)} .
\end{aligned}
$$


By inserting $w=1$ into (20), it follows that

$$
\Pi^{*}(z, 1)=\frac{\pi_{0} \lambda z(1-z)\left(1-S\left(\omega_{1}\right)\right)}{\eta \omega_{0}\left(1-S\left(\omega_{1}\right)\right)+\left(1-\omega_{1}\right)\left(S\left(\omega_{1}\right)-z\right)} .
$$

Let $P(z)$ denote the PGF of the system size distribution. From (16) and (21), we can obtain

$$
\begin{aligned}
P(z) & =\pi_{0}+\Psi^{*}(z, 1)+\Pi^{*}(z, 1) \\
& =\pi_{0}+\frac{\pi_{0} \lambda(1-z)(z+\eta E[R])\left(1-S\left(\omega_{1}\right)\right)}{\eta \omega_{0}\left(1-S\left(\omega_{1}\right)\right)+\left(1-\omega_{1}\right)\left(S\left(\omega_{1}\right)-z\right)},
\end{aligned}
$$

where $\pi_{0}=(\eta-\lambda(1-S(\bar{\eta}))) / \eta[1+\lambda E[R](1-S(\bar{\eta}))]$ by the normalizing condition (11).

Differentiating (22) with respect to $z$ and taking limit $z \rightarrow 1$ lead to the mean system size given by

$$
\begin{aligned}
L= & \frac{\lambda}{1+\lambda E[R](1-S(\bar{\eta}))} \\
& \cdot\left[\frac{1-S(\bar{\eta})}{\eta}\right. \\
& \left.+\frac{\bar{\eta} \lambda(1+\eta E[R])\left(1-S(\bar{\eta})-\eta S^{\prime}(\bar{\eta})\right)}{\eta(\eta-\lambda+\lambda S(\bar{\eta}))}\right],
\end{aligned}
$$

where $S^{\prime}(z)=d S(z) / d z$ in $(23)$.

Remark 1. System 1 is stable if and only if $|P(z)|<\infty$ for $|z| \leq 1$. The denominator of $P(z)$ for System 1 can be written as $\left(1-\omega_{1}\right)(A(z)-z)$, where

$$
A(z)=\frac{\eta \omega_{0}+\left(1-\omega_{0}\right) S\left(\omega_{1}\right)}{1-\omega_{1}} .
$$

Here, $\left(1-\omega_{1}\right)$ has no zeros for $|z| \leq 1$. By Rouché's theorem, $A(z)-z$ also has no zeros for $|z| \leq 1$ if and only if $d A(z) /\left.d z\right|_{z=1}<1$. Thus, the necessary and sufficient condition for the stability of System 1 is $\lambda<\eta(1-S(\bar{\eta}))^{-1}$.

3.2. Sojourn Time Distribution for System 1. We derive the PGF of the sojourn time of a test customer (TC) under the FCFS discipline for System 1, regardless of whether or not its service is interrupted by a negative customer. The sojourn time is defined as the sum of the queue waiting time and the service time. We do not take blocked customers' sojourn times into consideration because they are equal to 0 .

Let us define the actual service time as the actual amount of service time that a TC receives before departing the system either by a service completion or by a negative customer. Let $S_{a}$ and $S_{a}(z)$, respectively, denote the actual service time and its PGF under our assumption. Then, we obtain

$$
\begin{array}{r}
\operatorname{Pr}\left\{S_{a}=k\right\}=\operatorname{Pr}\{S=k\} \bar{\eta}^{k}+\operatorname{Pr}\{S \geq k\} \bar{\eta}^{k-1} \eta, \\
k \geq 1, \\
S_{a}(z)=\sum_{k=1}^{\infty} \operatorname{Pr}\left\{S_{a}=k\right\} z^{k}=\frac{\eta z+(1-z) S(\bar{\eta} z)}{1-\bar{\eta} z} .
\end{array}
$$

We further define the modified service time as the virtual service time, including a time to repair. Since a repair period follows each time a negative customer arrives, from the point of view of a TC, the modified service times can be regarded as service times of the customers who arrive prior to a TC. Let $S_{b}$ and $S_{b}(z)$, respectively, denote the modified service time and its PGF under our assumption. Then, we obtain

$$
\begin{gathered}
\operatorname{Pr}\left\{S_{b}=1\right\}=\operatorname{Pr}\{S=1\} \bar{\eta}, \\
\operatorname{Pr}\left\{S_{b}=k\right\}=\operatorname{Pr}\{S=k\} \bar{\eta}^{k}+\sum_{i=1}^{k-1} \operatorname{Pr}\{S \geq i\} \eta \bar{\eta}^{i-1} r_{k-i}, \\
k \geq 2, \\
S_{b}(z)=\sum_{k=1}^{\infty} \operatorname{Pr}\left\{S_{b}=k\right\} z^{k} \quad \\
=\frac{\eta z R(z)+(1-\bar{\eta} z-\eta z R(z)) S(\bar{\eta} z)}{1-\bar{\eta} z} .
\end{gathered}
$$

Since we assumed that during a slot the potential arrival of a negative customer and a repair completion occur immediately before the potential arrival of a positive customer, a TC's arrival which occurs during $\left(t^{-}, t\right)$ may belong to one of the following cases.

Case 1. A TC arrives finding the server is idle. Then, the TC's service is immediately started.

Case 2. A TC arrives at a slot during which a repair completion occurs. Then, the waiting time of the TC is the total service time of the customers who are in the queue on the arrival of the TC because the repair has just been completed.

Case 3. A TC arrives at a slot during which the server is busy and a negative customer does not arrive. Then, the waiting time of the TC is the total service time of the customers who are in the queue on the arrival of the TC, plus the remaining modified service time of the customer in service.

In other cases, a positive customer is blocked, and we do not take blocked customers into consideration for waiting time distribution. Let $W_{q, i}$ denote the waiting time of a TC that arrives in case $i, i=1,2,3$, and define $W_{q, i}(z)=$ $\operatorname{Pr}\{$ Case $i\} E\left[z^{W_{q, i}} \mid\right.$ Case $\left.i\right]$. From Case 1, we have

$$
\begin{aligned}
& W_{q, 1}(z) \\
& \quad=\lim _{t \rightarrow \infty} \operatorname{Pr}\left\{N\left(t^{-}\right)=0, \xi\left(t^{-}\right)=1\right\} z^{0} \\
& =\lim _{t \rightarrow \infty} \operatorname{Pr}\left\{N\left((t-1)^{+}\right)=0, \xi\left((t-1)^{+}\right)=1\right\} \\
& =\pi_{0} .
\end{aligned}
$$

Case 2 yields

$$
\begin{aligned}
& W_{q, 2}(z) \\
& =\lim _{t \rightarrow \infty} \sum_{n=0}^{\infty} \operatorname{Pr}\left\{N\left(t^{-}\right)=n, \xi\left(t^{-}\right)=0, \xi\left(t^{+}\right)=1\right\}\left[S_{b}(z)\right]^{n}
\end{aligned}
$$




$$
\begin{aligned}
& =\lim _{t \rightarrow \infty} \sum_{n=0}^{\infty} \operatorname{Pr}\left\{N\left((t-1)^{+}\right)=n, R_{R}\left((t-1)^{+}\right)=1\right\}\left[S_{b}(z)\right]^{n} \\
& =\sum_{n=0}^{\infty} \psi_{n}(1)\left[S_{b}(z)\right]^{n}=\Psi\left(S_{b}(z), 1\right) .
\end{aligned}
$$

From Case 3, we have

$$
\begin{aligned}
& W_{q, 3}(z) \\
& =\lim _{t \rightarrow \infty} \sum_{n=1}^{\infty} \sum_{k=0}^{\infty} \operatorname{Pr}\left\{N\left(t^{-}\right)=n, S_{R}\left(t^{-}\right)=k,\right. \\
& \left.\xi\left(t^{-}\right)=1, \xi\left(t^{+}\right)=1\right\}\left[S_{b}(z)\right]^{n-1} \\
& \times\left[\operatorname{Pr}\left\{S_{R}\left(t^{-}\right)<Y \mid S_{R}\left(t^{-}\right)=k\right\} z^{k}\right. \\
& \left.+\sum_{h=1}^{k} \operatorname{Pr}\left\{Y=h \mid S_{R}\left(t^{-}\right)=k\right\} z^{h} R(z)\right] \\
& =\lim _{t \rightarrow \infty} \sum_{n=1}^{\infty} \sum_{k=0}^{\infty} \operatorname{Pr}\left\{N\left((t-1)^{+}\right)=n, S_{R}\left((t-1)^{+}\right)=k+1,\right. \\
& \left.\quad \xi\left((t-1)^{+}\right)=1\right\} \bar{\eta}\left[S_{b}(z)\right]^{n-1} \\
& \quad+\frac{\eta}{S_{b}(z)}\left[z^{-1} \Pi^{*}\left(S_{b}(z), \bar{\eta} z\right)\right. \\
& \left.+\bar{\eta} \sum_{n=1}^{\infty} \sum_{k=1}^{\infty} \pi_{n}(k)\left[S_{b}(z)\right]^{n-1} R(z) \frac{\eta z\left(1-(\bar{\eta} z)^{k-1}\right)}{1-\bar{\eta} z} R\left(S_{b}(z), 1\right)-z^{-1} \Pi^{*}\left(S_{b}(z), \bar{\eta} z\right)\right) \\
& \left.+\sum_{h=1}^{\infty} \sum_{k=1}^{\infty} \pi_{n}(k)\left[S_{b}(z)\right]^{n-1}(\bar{\eta} z)^{k-1} \operatorname{Pr}\left\{Y=h \mid S_{R}\left(t^{-}\right)=k\right\} z^{h} R(z)\right] \\
& =\bar{\eta} \sum_{n=1}^{\infty} \sum_{k=0}^{\infty} \pi_{n}(k+1)\left[S_{b}(z)\right]^{n-1}\left[\bar{\eta}^{k} z^{k}+\sum_{h=1}^{k} \bar{\eta}{ }^{h-1} \eta z^{h} R(z)\right]
\end{aligned}
$$

Let $W_{q}$ denote the unconditional waiting time of a TC. Combining (27a), (27b), and (27c) with (14), we have $W_{q}(z)$ given by

$$
\begin{aligned}
W_{q}(z)= & \frac{W_{q, 1}(z)+W_{q, 2}(z)+W_{q, 3}(z)}{W_{q, 1}(1)+W_{q, 2}(1)+W_{q, 3}(1)} \\
= & \frac{1}{1-P_{R}} \\
& \cdot\left[\pi_{0}+\frac{\Pi^{*}\left(S_{b}(z), \bar{\eta} z\right)}{z S_{b}(z)}\left(1-\frac{\eta z}{1-\bar{\eta} z} R(z)\right)\right. \\
& \left.\quad \frac{\Pi^{*}\left(S_{b}(z), 1\right)}{S_{b}(z)}\left(\eta+\bar{\eta} \frac{\eta z}{1-\bar{\eta} z} R(z)\right)\right],
\end{aligned}
$$

where $P_{R}=\lambda E[R](1-S(\bar{\eta})) /(1+\lambda E[R](1-S(\bar{\eta})))$, which denotes the probability that the arrival of a positive customer is blocked.

Remark 2. Now that we deal with the Bernoulli arrival process queueing system, BASTA property [22] is employed to derive $W_{q, i}(z)$.

Let $W$ be a TC's sojourn time. As $W_{q}$ and $S_{a}$ are independent, we finally obtain

$$
W(z)=W_{q}(z) \cdot S_{a}(z),
$$

which leads to the mean sojourn time given by

$$
\begin{aligned}
E[W]= & \frac{1-S(\bar{\eta})}{\eta} \\
& +\frac{\bar{\eta} \lambda(1+\eta E[R])\left(1-S(\bar{\eta})-\eta S^{\prime}(\bar{\eta})\right)}{\eta(\eta-\lambda+\lambda S(\bar{\eta}))} .
\end{aligned}
$$

Remark 3. Equations (23) and (30) confirm the result of Little's law: $L=\lambda_{e} E[W]$, where the effective arrival rate $\lambda_{e}=\lambda\left(1-P_{R}\right)$.

Remark 4. Since $P_{R}$ is the blocking probability of a positive customer, the service canceling probability $P_{C}$ of a positive customer is given by $P_{C}=\left(1-P_{R}\right)(1-S(\bar{\eta}))$ because a servicecanceled positive customer first enters the system without blocking and then a negative customer arrives before the completion of the service of the service-canceled positive customer.

\section{System 2}

In System 2, newly arriving customers enter the system while the server is under repair. They receive their service after repair. 
4.1. System Size Distribution for System 2. With the limiting probabilities defined in Section 2, the Kolmogorov equations that govern System 2 can be written as follows:

$$
\begin{gathered}
\psi_{0}(k)=\psi_{0}(k+1) \bar{\lambda}+\eta \pi_{1} \bar{\lambda} r_{k}, \\
\psi_{n}(k)=\psi_{n-1}(k+1) \lambda+\psi_{n}(k+1) \bar{\lambda}+\eta \pi_{n} \lambda r_{k} \\
+\eta \pi_{n+1} \bar{\lambda} r_{k}, \quad n \geq 1, \\
\pi_{0}=\left(\psi_{0}(1)+\pi_{0}+\bar{\eta} \pi_{1}(1)\right) \bar{\lambda}, \\
\pi_{1}(k)=\psi_{0}(1) \lambda s_{k}+\psi_{1}(1) \bar{\lambda} s_{k}+\pi_{0} \lambda s_{k} \\
+\bar{\eta}\left(\pi_{1}(1) \lambda s_{k}+\pi_{1}(k+1) \bar{\lambda}+\pi_{2}(1) \bar{\lambda} s_{k}\right), \\
\pi_{n}(k)=\psi_{n-1}(1) \lambda s_{k}+\psi_{n}(1) \bar{\lambda} s_{k} \\
+\bar{\eta}\left(\pi_{n-1}(k+1) \lambda+\pi_{n}(1) \lambda s_{k}\right. \\
\left.+\pi_{n}(k+1) \bar{\lambda}+\pi_{n+1}(1) \bar{\lambda} s_{k}\right), \quad n \geq 2,
\end{gathered}
$$

and the normalizing condition is given by

$$
\pi_{0}+\Psi^{*}(1,1)+\Pi^{*}(1,1)=1
$$

Multiplying (31) and (32) by $z^{n}$ and summing over $n, n \geq$ 0 , we obtain

$$
\Psi(z, k)=\omega_{0}\left(\Psi(z, k+1)+\eta r_{k} z^{-1} \Pi^{*}(z, 1)\right),
$$

where $\omega_{0}=\bar{\lambda}+\lambda z$. Multiplying (38) by $w^{k}$ and summing over $k, k \geq 1$, yield

$$
\begin{aligned}
\Psi^{*} & (z, w)\left(1-w^{-1} \omega_{0}\right) \\
& =\omega_{0}\left(\eta z^{-1} R(w) \Pi^{*}(z, 1)-\Psi(z, 1)\right) .
\end{aligned}
$$

Inserting $w=\omega_{0}$ into (38) and solving $\Psi(z, 1)$, we obtain

$$
\Psi(z, 1)=\eta z^{-1} R\left(\omega_{0}\right) \Pi^{*}(z, 1) .
$$

Substituting (39) into (38) results in

$$
\Psi^{*}(z, w)=\frac{\eta w \omega_{0}\left(R(w)-R\left(\omega_{0}\right)\right)}{z\left(w-\omega_{0}\right)} \Pi^{*}(z, 1) .
$$

Letting $w=1$ in (40), we obtain the relationship

$$
\Psi^{*}(z, 1)=\frac{\eta \omega_{0}\left(1-R\left(\omega_{0}\right)\right)}{z\left(1-\omega_{0}\right)} \Pi^{*}(z, 1) .
$$

Multiplying (34) and (35) by $z^{n}$ and then summing over $n, n \geq 1$, together with (33), we obtain

$$
\begin{aligned}
& \Pi(z, k) \\
& \quad=\omega_{1} \Pi(z, k+1) \\
& \quad+s_{k}\left[\omega_{0} \Psi(z, 1)+z^{-1} \omega_{1} \Pi(z, 1)-\pi_{0} \lambda(1-z)\right],
\end{aligned}
$$

where $\omega_{1}=\bar{\eta}(\bar{\lambda}+\lambda z)$. Multiplying (42) by $w^{k}$ and summing over $k, k \geq 1$, yield

$$
\begin{aligned}
& \Pi^{*}(z, w)\left(1-w^{-1} \omega_{1}\right) \\
& =z^{-1} \omega_{1} \Pi(z, 1)(S(w)-z) \\
& \quad+S(w)\left[\omega_{0} \Psi(z, 1)-\pi_{0} \lambda(1-z)\right] .
\end{aligned}
$$

To solve $\Pi(z, 1)$, we insert $w=\omega_{1}$ into (43). Thus, we have

$$
\Pi(z, 1)=\frac{z S\left(\omega_{1}\right)\left[\pi_{0} \lambda(1-z)-\omega_{0} \Psi(z, 1)\right]}{\omega_{1}\left(S\left(\omega_{1}\right)-z\right)} .
$$

Using (39) and (44) in (43), $\Pi^{*}(z, w)$ is represented as

$$
\begin{aligned}
& \Pi^{*}(z, w) \\
& =\frac{w\left(S(w)-S\left(\omega_{1}\right)\right)\left(\pi_{0} \lambda z(1-z)-\eta \omega_{0} R\left(\omega_{0}\right) \Pi^{*}(z, 1)\right)}{\left(w-\omega_{1}\right)\left(S\left(\omega_{1}\right)-z\right)} .
\end{aligned}
$$

By inserting $w=1$ into (45), it follows that

$$
\begin{aligned}
& \Pi^{*}(z, 1) \\
& \quad=\frac{\pi_{0} \lambda z(1-z)\left(1-S\left(\omega_{1}\right)\right)}{\eta \omega_{0} R\left(\omega_{0}\right)\left(1-S\left(\omega_{1}\right)\right)+\left(1-\omega_{1}\right)\left(S\left(\omega_{1}\right)-z\right)} .
\end{aligned}
$$

Let $P(z)$ denote the PGF of the system size distribution. From (41) and (46), we can obtain

$$
\begin{aligned}
P(z) & =\pi_{0}+\Psi^{*}(z, 1)+\Pi^{*}(z, 1) \\
& =\pi_{0}+\frac{\pi_{0}\left(1-S\left(\omega_{1}\right)\right)\left[\lambda z(1-z)+\eta \omega_{0}\left(1-R\left(\omega_{0}\right)\right)\right]}{\eta \omega_{0} R\left(\omega_{0}\right)\left(1-S\left(\omega_{1}\right)\right)+\left(1-\omega_{1}\right)\left(S\left(\omega_{1}\right)-z\right)},
\end{aligned}
$$

where $\pi_{0}=(\eta-\lambda(1+\eta E[R])(1-S(\bar{\eta}))) / \eta$ by the normalizing condition (36).

Differentiating (47) with respect to $z$ and taking limit $z \rightarrow 1$ lead to the mean system size given by

$$
\begin{aligned}
& L= \frac{\lambda(1-S(\bar{\eta}))}{\eta} \\
&+\left(\left(\lambda ^ { 2 } \left[\left(2 \bar{\eta}+2 \eta E[R]+\eta^{2} E[R(R+1)]\right)\right.\right.\right. \\
&\left.\left.\cdot(1-S(\bar{\eta}))-2 \eta \bar{\eta}(1+\eta E[R]) S^{\prime}(\bar{\eta})\right]\right) \\
&\left.\cdot(2 \eta[\eta-\lambda(1+\eta E[R])(1-S(\bar{\eta}))])^{-1}\right) .
\end{aligned}
$$

Remark 5. The denominator of $P(z)$ for System 2 can be written as $\left(1-\omega_{1}\right)(B(z)-z)$, where

$$
B(z)=\frac{\eta \omega_{0} R\left(\omega_{0}\right)+\left(1-\omega_{1}-\eta \omega_{0} R\left(\omega_{0}\right)\right) S\left(\omega_{1}\right)}{1-\omega_{1}} .
$$

Similar to Remark 1, for the stability of System 2, the following inequality is the necessary and sufficient condition under which steady-state solution exists: $\lambda<\eta[(1+\eta E[R])(1-$ $S(\bar{\eta}))]^{-1}$. 
4.2. Sojourn Time Distribution for System 2. We derive the PGF of the FCFS sojourn time of a TC for System 2. The method used in this section is much simpler than that used in Section 3.2. The PGF of the waiting time for the standard $\mathrm{Geo} / \mathrm{G} / 1$ queue is known to be [22]

$$
\frac{c(1-z)}{1-z-\lambda+\lambda S(z)}
$$

where $c$ is a normalizing constant. We first replace $S(z)$ in (50) with $S_{b}(z)$ in (27a), (27b), and (27c). Then, $c$ is determined by normalization. Applying this procedure, $W_{q}(z)$ is given by

$$
\begin{aligned}
& W_{q}(z) \\
& =\frac{c(1-z)(1-\bar{\eta} z)}{(1-z)(1-\bar{\eta} z)-\lambda(1-\bar{\eta} z-\eta z R(z))(1-S(\bar{\eta} z))},
\end{aligned}
$$

where $c=(\eta-\lambda(1+\eta E[R])(1-S(\bar{\eta}))) / \eta$. Since $W_{q}$ and $S_{a}$ are independent, we have $W(z)$ as

$$
W(z)=W_{q}(z) \cdot S_{a}(z)
$$

which leads to the mean sojourn time given by

$$
\begin{aligned}
& E[W] \\
& =\frac{1-S(\bar{\eta})}{\eta} \\
& +\left(\left(\lambda \left[\left(2 \bar{\eta}+2 \eta E[R]+\eta^{2} E[R(R+1)]\right)\right.\right.\right. \\
& \left.\left.\cdot(1-S(\bar{\eta}))-2 \eta \bar{\eta}(1+\eta E[R]) S^{\prime}(\bar{\eta})\right]\right) \\
& \left.\cdot(2 \eta[\eta-\lambda(1+\eta E[R])(1-S(\bar{\eta}))])^{-1}\right) .
\end{aligned}
$$

Remark 6. Equations (48) and (53) confirm the result of Little's law: $L=\lambda E[W]$.

Remark 7. The service canceling probability $P_{C}$ of a positive customer is given by $P_{C}=1-S(\bar{\eta})$ because a negative customer arrives before the completion of the service of a service-canceled positive customer.

\section{Numerical Examples}

The purpose of this section is to study the influence of negative customer arrival on the mean system size and the service canceling probability of both System 1 and System 2. We also investigate the influence of the type of the repair time distribution on the mean system size. In every example, positive customers arrive at a system, according to a Bernoulli process at a rate of 0.1 . We consider three types of service time distributions: a geometric distribution, a negative binomial distribution, and a mixture of two different geometric distributions. Specifically, for the geometric case, the probability mass function (PMF) of the service time is defined by

$$
s_{k}=\left(\frac{1}{8}\right)\left(\frac{7}{8}\right)^{k-1}, \quad k=1,2, \ldots
$$

For the negative binomial case, the PMF of the service time is defined by

$$
s_{k}=\left(\begin{array}{c}
k-1 \\
3
\end{array}\right)\left(\frac{1}{2}\right)^{4}\left(\frac{1}{2}\right)^{k-4}, \quad k=4,5, \ldots
$$

For the geometric mixture case, the PMF of the service time is defined by

$$
\begin{array}{r}
s_{k}=\frac{4}{5}\left(\frac{1}{3}\right)\left(\frac{2}{3}\right)^{k-1}+\frac{1}{5}\left(\frac{1}{28}\right)\left(\frac{27}{28}\right)^{k-1}, \\
k=1,2, \ldots .
\end{array}
$$

The above three service times have a common mean of 8 . The coefficients of variation are, respectively, $0.94,0.35$, and 2 for the geometric, negative binomial, and geometric mixture cases.

Similarly, the repair time distributions are assumed to follow one of the three distributions. For the geometric case, the PMF of the repair time is defined by

$$
r_{k}=\left(\frac{1}{5}\right)\left(\frac{4}{5}\right)^{k-1}, \quad k=1,2, \ldots
$$

For the negative binomial case, the PMF of the repair time is defined by

$$
r_{k}=\left(\begin{array}{c}
k-1 \\
1
\end{array}\right)\left(\frac{2}{5}\right)^{2}\left(\frac{3}{5}\right)^{k-2}, \quad k=2,3, \ldots
$$

For the geometric mixture case, the PMF of the repair time is defined by

$$
\begin{aligned}
r_{k}= & \frac{7}{10}\left(\frac{3}{20}\right)\left(\frac{17}{20}\right)^{k-1} \\
& +\frac{3}{10}\left(\frac{9}{10}\right)\left(\frac{1}{10}\right)^{k-1}, \quad k=1,2, \ldots
\end{aligned}
$$

While these three repair times have a common mean of 5 , their coefficients of variation are, respectively, $0.89,0.55$, and 1.15 for the geometric, negative binomial, and geometric mixture cases.

In Figures 1, 2, and 3, the mean system sizes and the service canceling probabilities of both System 1 and System 2 are shown as functions of $\eta$ when the repair time follows a negative binomial distribution.

Figures 1 to 3 confirm that the mean system sizes of both System 1 and System 2 decrease as $\eta$ increases. Furthermore, for all cases, the mean system size of System 2 is greater than the mean system size of System 1 as expected. This is obvious because, in System 2, customers newly arriving during the repair period join the queue and wait for the server to be repaired without leaving the system. We also verify that the service canceling probabilities of both System 1 and System 2 increase as $\eta$ increases and the service canceling probability of System 2 is greater than that of System 1 for all cases. This is because, in System 1, the arrival of a positive customer can be blocked. 


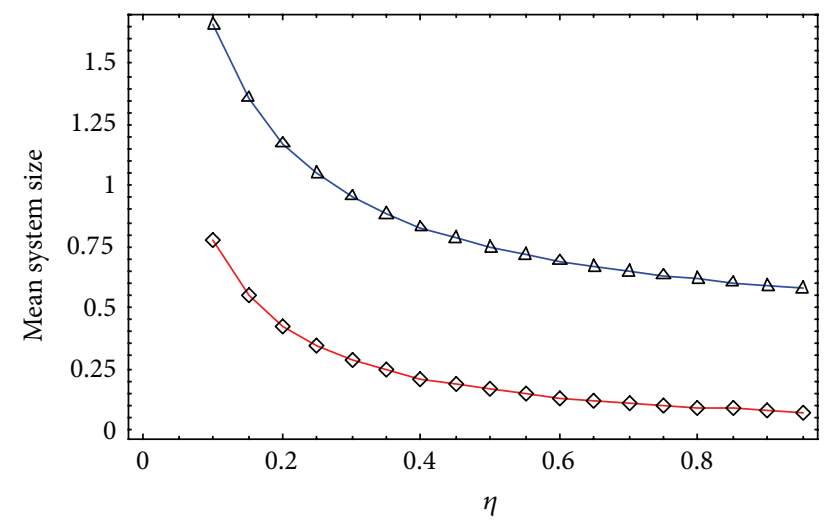

Geo service $\diamond$ System 1 $\triangle$ System 2

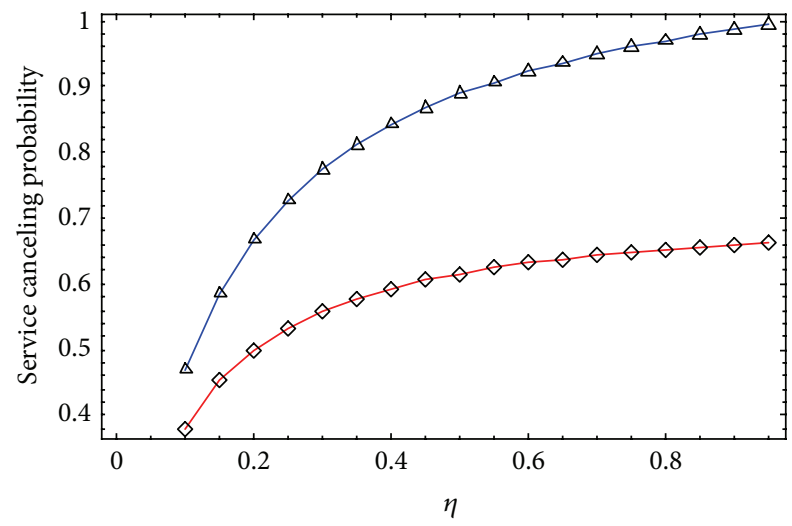

Geo service

$\diamond$ System 1

$\triangle$ System 2

FIGURE 1: Mean system size and service canceling probability over $\eta$ with geometric service time distribution.

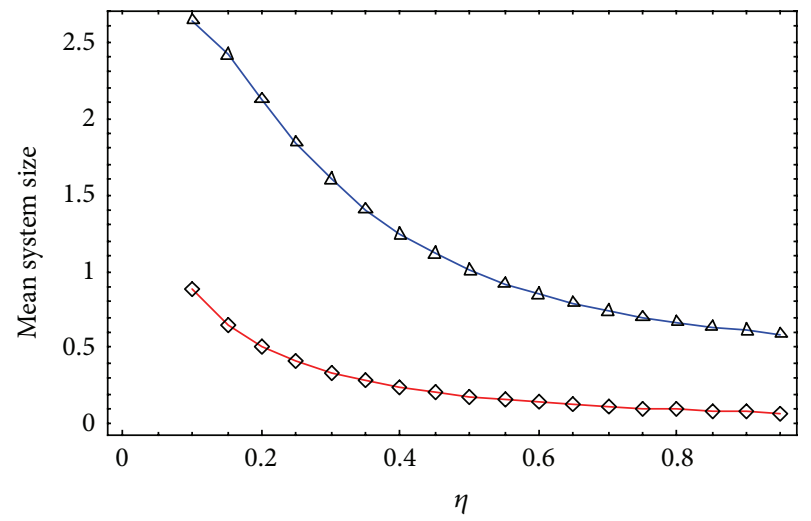

Negative binomial service $\diamond$ System 1

$\triangle$ System 2

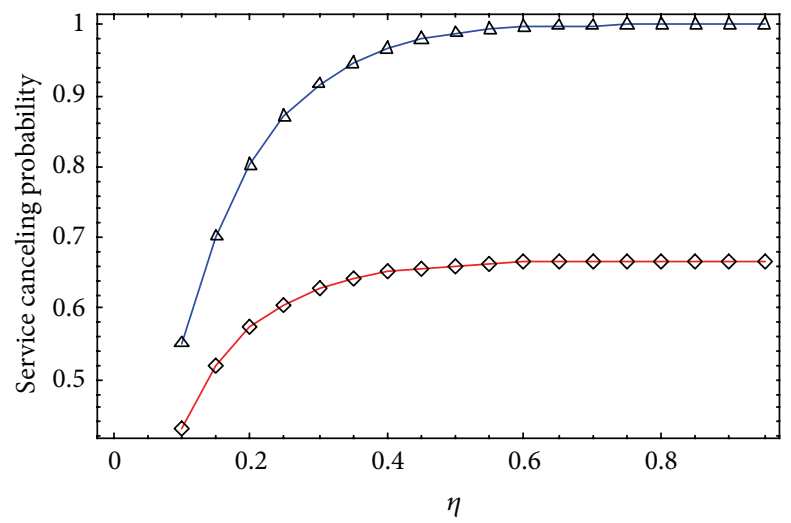

Negative binomial service $\diamond$ System 1 $\triangle$ System 2

FIGURE 2: Mean system size and service canceling probability over $\eta$ with negative binomial service time distribution.

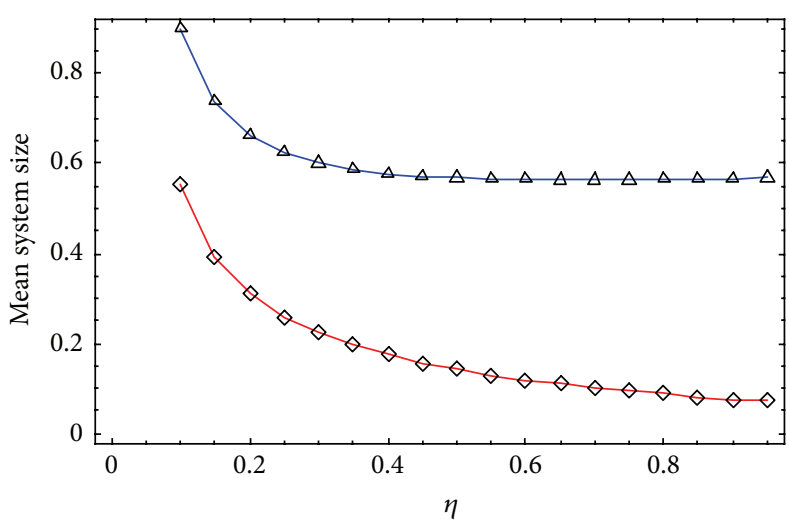

Geometric mixture service $\diamond$ System 1

$\triangle$ System 2

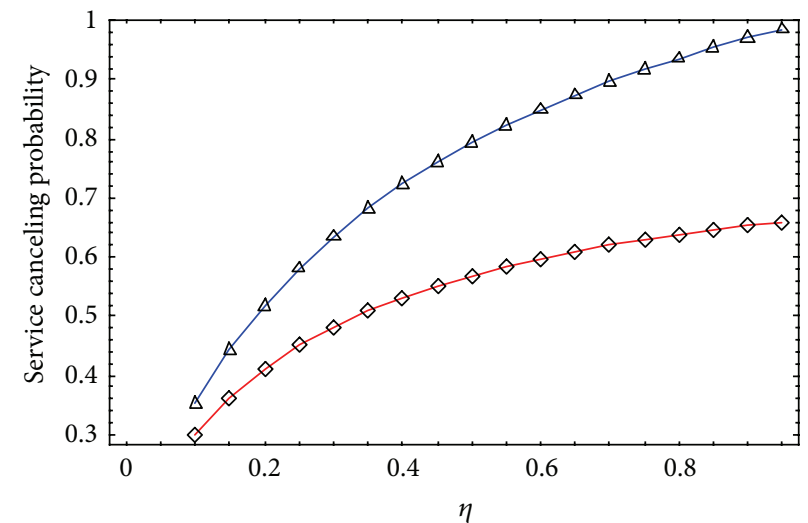

Geometric mixture service $\diamond$ System 1 $\triangle$ System 2

FIGURE 3: Mean system size and service canceling probability over $\eta$ with geometric mixture service time distribution. 


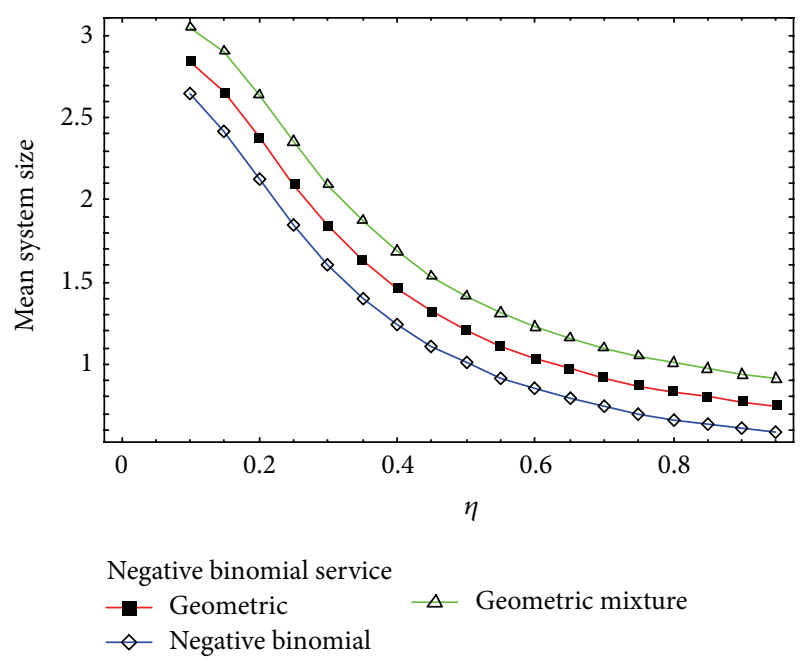

FIGURE 4: Mean system size over $\eta$ with various repair time distributions.

Second, we investigate the tendencies of the mean system size of System 2 by varying the repair time distributions. In Figure 4, the mean system size of System 2 is shown as a function of $\eta$ when the service time follows the negative binomial distribution.

As shown in Figure 4, for all three different distributions of repair times, as $\eta$ increases, the mean system sizes decrease. Moreover, for all values of $\eta$, the higher the coefficient of variation is, the greater the mean system size is. The overall results in this section confirm that the mean system size is a decreasing function of $\eta$.

\section{Conclusion}

We considered two repairable Geo/G/1 queueing systems with negative customers, in which a negative customer arrival causes the customer in service to leave the system and the server to fail. At a failure epoch, the repair process immediately begins. Those arriving during the repair period are blocked in System 1, while they remain in System 2. For these systems, we derived some important results of system characteristics such as the PGFs of the system sizes and FCFS sojourn times. Finally, some numerical examples were performed to illustrate the influence of the negative customer arrival rate on the systems. Our research presents an extension of the discrete-time repairable queueing model and the analysis of the model may provide a decision making tool for repair policies arising in many practical systems.

For further studies, we can extend this model to more complex situations such as the discrete-time repairable queueing systems with the phase type arrival process of either positive customers or negative customers.

\section{Conflict of Interests}

The authors declare that there is no conflict of interests regarding the publication of this paper.

\section{References}

[1] E. Gelenbe, "Random neural networks with negative and positive signals and product form solution," Neural Computation, vol. 1, no. 4, pp. 502-510, 1989.

[2] R. J. Boucherie and O. J. Boxma, "The workload in the M/G/1 queue with work removal," Probability in the Engineering and Informational Sciences, vol. 10, no. 2, pp. 261-277, 1996.

[3] C. Morfopoulou, "Network routing control with G-networks," Performance Evaluation, vol. 68, no. 4, pp. 320-329, 2011.

[4] J. Wu and X. Yin, "An M/G/1 retrial G-Queue with non-exhaustive random vacations and an unreliable server," Computers \& Mathematics with Applications, vol. 62, no. 5, pp. 2314-2329, 2011.

[5] P. G. Harrison and E. Pitel, "Sojourn times in single-server queues with negative customers," Journal of Applied Probability, vol. 30, no. 4, pp. 943-963, 1993.

[6] P. G. Harrison and E. Pitel, “ The M/G/1 queue with negative customers," Advances in Applied Probability, vol. 28, no. 2, pp. 540-566, 1996.

[7] W. S. Yang and K. C. Chae, "A note on the GI/M/1 queue with Poisson negative arrivals," Journal of Applied Probability, vol. 38, no. 4, pp. 1081-1085, 2001.

[8] J. R. Artalejo, "G-networks: a versatile approach for work removal in queueing networks," European Journal of Operational Research, vol. 126, no. 2, pp. 233-249, 2000.

[9] T. V. Do, "An initiative for a classified bibliography on G-networks," Performance Evaluation, vol. 68, no. 4, pp. 385-394, 2011.

[10] T. V. Do, "Bibliography on G-networks, negative customers and applications," Mathematical and Computer Modelling, vol. 53, no. 1-2, pp. 205-212, 2011.

[11] E. Gelenbe, "Product-form queueing networks with negative and positive customers," Journal of Applied Probability, vol. 28, no. 3, pp. 656-663, 1991.

[12] E. Gelenbe, "G-networks: a unifying model for neural and queueing networks," Annals of Operations Research, vol. 48, no. 5, pp. 433-461, 1994.

[13] E. Gelenbe, “The first decade of G-networks," European Journal of Operational Research, vol. 126, no. 2, pp. 231-232, 2000.

[14] I. Atencia and P. Moreno, "A single-server G-queue in discretetime with geometrical arrival and service process," Performance Evaluation, vol. 59, no. 1, pp. 85-97, 2005.

[15] W. H. Zhou, "Performance analysis of discrete-time queue GI/G/1 with negative arrivals," Applied Mathematics and Computation, vol. 170, no. 2, pp. 1349-1355, 2005.

[16] J. Wang and P. Zhang, "A discrete-time retrial queue with negative customers and unreliable server," Computers and Industrial Engineering, vol. 56, no. 4, pp. 1216-1222, 2009.

[17] H. M. Park, W. S. Yang, and K. C. Chae, "The Geo/G/1 queue with negative customers and disasters," Stochastic Models, vol. 25, no. 4, pp. 673-688, 2009.

[18] K. C. Chae, H. M. Park, and W. S. Yang, "A GI/Geo/1 queue with negative and positive customers," Applied Mathematical Modelling, vol. 34, no. 6, pp. 1662-1671, 2010.

[19] J. Wu, Z. Liu, and Y. Peng, "On the BMAP/G/1 G-queues with second optional service and multiple vacations," Applied Mathematical Modelling, vol. 33, no. 12, pp. 4314-4325, 2009.

[20] J. Wu, Z. Liu, and G. Yang, "Analysis of the finite source \$MAP/ $\mathrm{PH} / \mathrm{N}$ \$ retrial \$G\$-queue operating in a random environment," Applied Mathematical Modelling: Simulation and Computation for Engineering and Environmental Systems, vol. 35, no. 3, pp. 1184-1193, 2011. 
[21] D. H. Lee, W. S. Yang, and H. M. Park, "Geo/G/1 queues with disasters an d general repair times," Applied Mathematical Modelling, vol. 35, no. 4, pp. 1561-1570, 2011.

[22] H. Takagi, Queueing Analysis: A Foundation of Performance Evaluation. Vol. 3, North-Holland, Amsterdam, The Netherlands, 1993. 


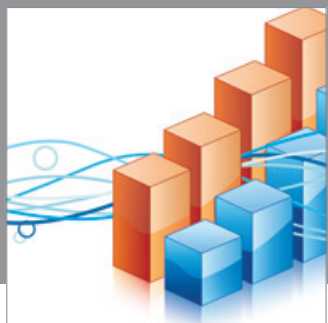

Advances in

Operations Research

mansans

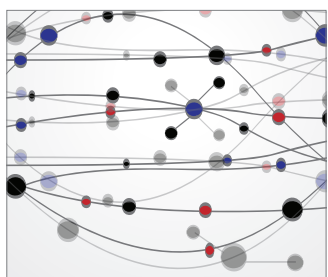

The Scientific World Journal
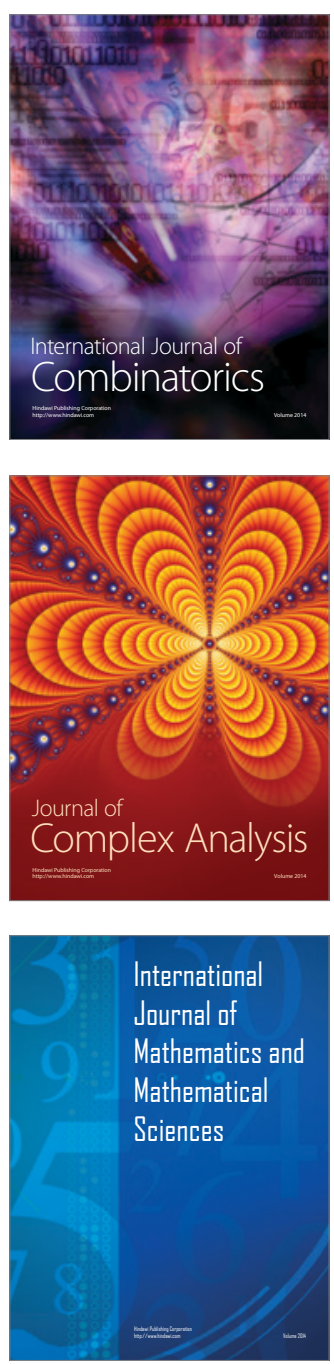
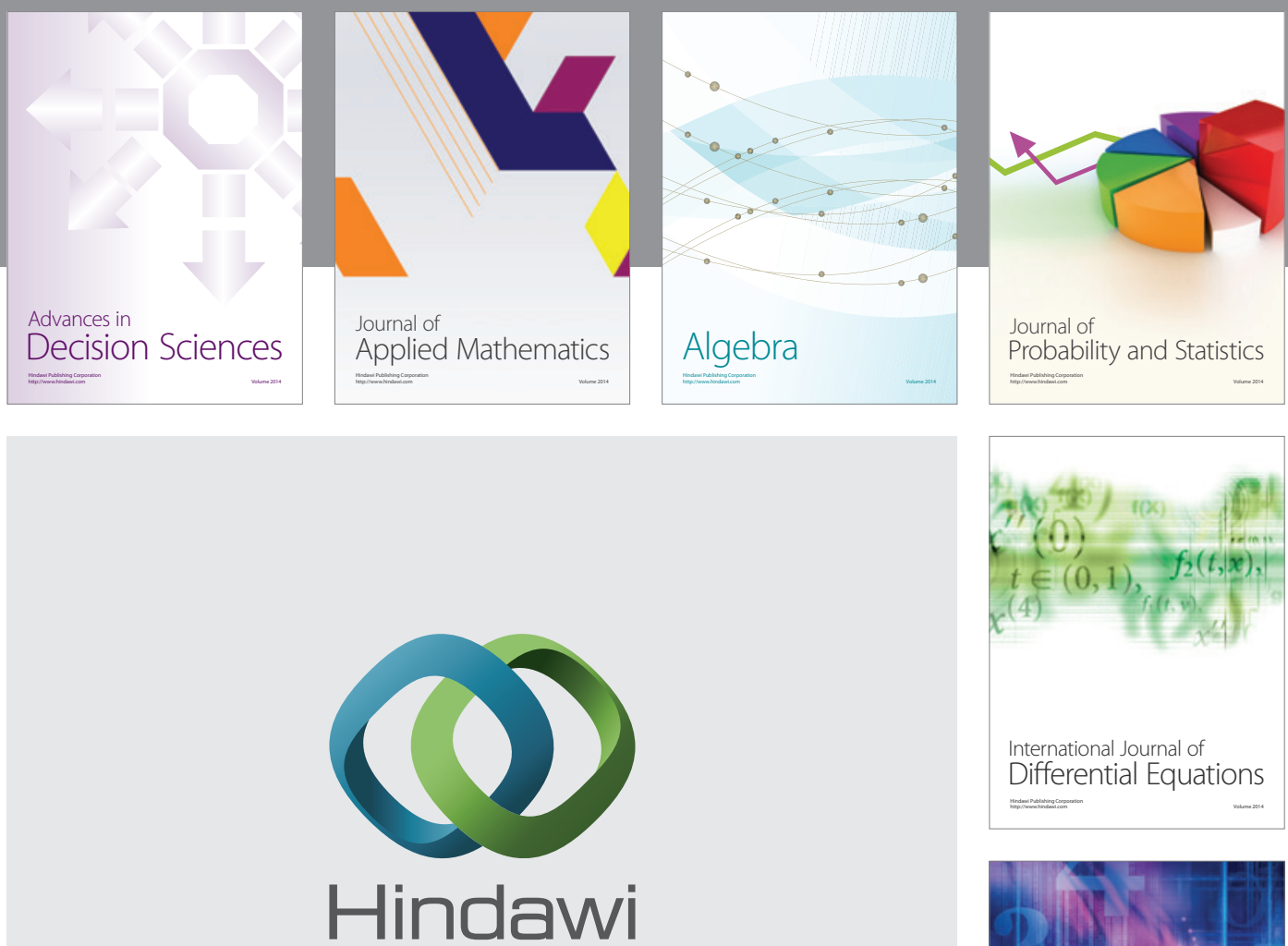

Submit your manuscripts at http://www.hindawi.com
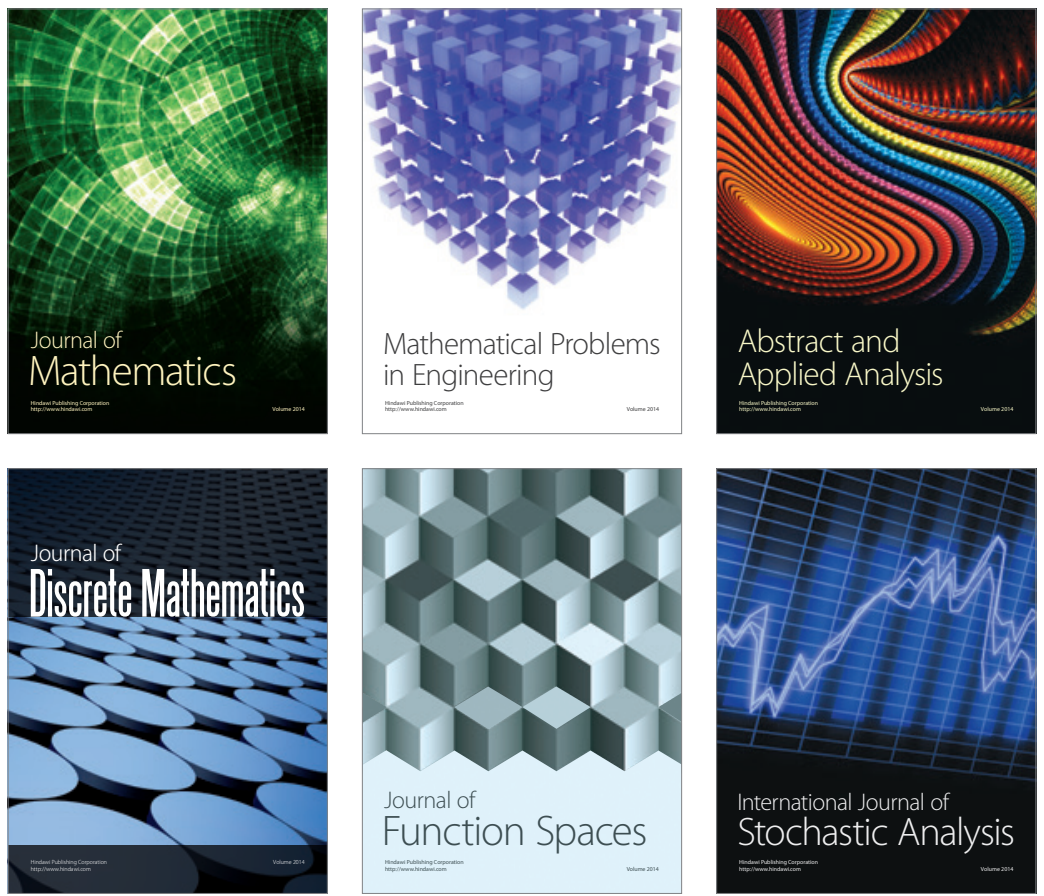

Journal of

Function Spaces

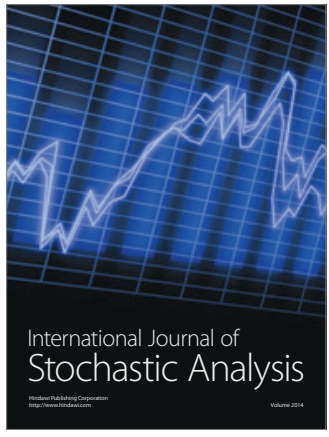

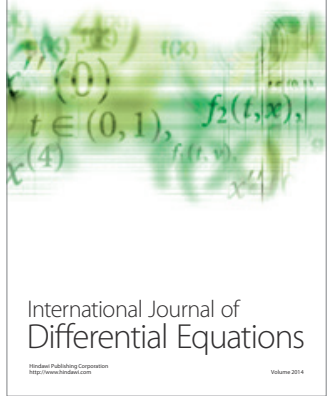
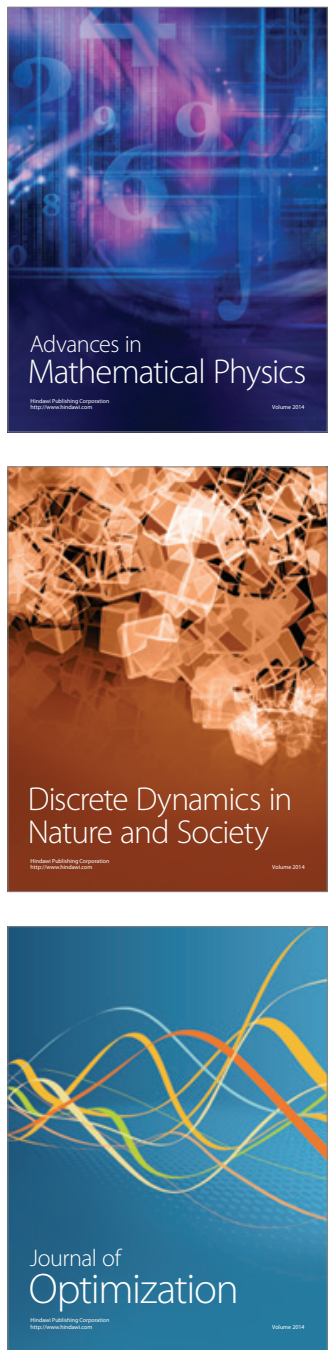